\title{
Characterization of fractional-quantum-Hall-effect quasiparticles
}

\author{
Alfred S. Goldhaber ${ }^{a, b, f}$ and J.K. Jain ${ }^{a, f}$ \\ ${ }^{a}$ Department of Physics and ${ }^{b}$ Institute for Theoretical Physics, State University of \\ New York, \\ Stony Brook, New York 11794-3800, USA.
}

August 7, 2018

\begin{abstract}
Composite fermions in a partially filled quasi-Landau level may be viewed as quasielectrons of the underlying fractional quantum Hall state, suggesting that a quasielectron is simply a dressed electron, as often is true in other interacting electron systems, and as a result has the same intrinsic charge and exchange statistics as an electron. This paper discusses how this result is reconciled with the earlier picture in which quasiparticles are viewed as fractionally-charged fractional-statistics "solitons". While the two approaches provide the same answers for the long-range interactions between the quasiparticles, the dressed-electron description is more conventional and unifies the view of quasiparticle dynamics in and beyond the fractional quantum Hall regime.
\end{abstract}

\footnotetext{
${ }^{1}$ goldhab@insti.physics.sunysb.edu

2 jain@insti.physics.sunysb.edu
} 


\section{INTRODUCTION}

An intriguing feature of the fractional quantum Hall effect (FQHE) [1] is the existence of two theoretical perspectives, one formulated in terms of fractionallycharged fractional-statistics quasiparticles [2, 3, 4, and the other in terms of charge $-e$ fermions, called composite fermions [5]. Such a circumstance occurs often in physics, and experience shows that we can gain insight by exploring all consistent perspectives, since for particular purposes one or another may be advantageous. The purpose of this note is to analyze the different ways in which the two approaches mentioned above obtain the same results for a set of quantities characterizing the topological properties of a given incompressible FQHE state. The considerations below are simple and straightforward, but to the best of our knowledge have not been spelled out clearly in the literature.

In both descriptions, Laughlin's elegant and successful wave function has played a crucial role: At filling factor $\nu=1 /(2 m+1)$, where $m$ is an integer, the Laughlin ground state [2] is

$$
\chi_{L}=\prod_{j<k}\left(z_{j}-z_{k}\right)^{2 m+1} \exp \left[-\frac{1}{4} \sum_{i}\left|z_{i}\right|^{2}\right],
$$

where $z_{j}=x_{j}-i y_{j}$ denotes the position of the $j$ th electron. The picture which arises from this wave function is one in which the quasiparticles (we use the word 'quasiparticle' to denote either a 'quasielectron' or a 'quasihole') are considered as topological solitons, carrying fractional electric charge $\left|e^{*}\right|=e /(2 m+1)$ [2]. Further, they are anyons, i.e., when one quasiparticle moves halfway around another, this produces a fractional 'statistics phase' $\pi \theta$, where $\theta=1 /(2 m+1)$ [3, 6]. To describe filling factors other than $\nu=1 /(2 m+1)$, the quasiparticle-hierarchy (QPH) approach starts with a 'parent' state, and builds Laughlin states out of its fractionally-charged fractional-statistics quasiparticles to produce 'daughter' states [3, 4]. An iteration of this procedure obtains the possibility of FQHE at all odd-denominator fractions starting from the Laughlin states at $\nu=1 /(2 m+1)$. We consider here only the principal fractions $n /(2 m n+1)$. The charge of the quasiparticle of this state is given by [2, 3, 4]

$$
\left|e^{*}\right|=\frac{e}{2 m n+1}
$$

and the "anyon statistics" by [3]

$$
\theta=\frac{2 m(n-1)+1}{2 m n+1},
$$

defined so that a complete loop of one quasiparticle around another produces a phase factor $e^{i 2 \pi \theta}$. (This definition of statistics has to do with the dynamical phase acquired by the wave function as quasiparticles wind around each other. We call it "anyon statistics" to distinguish it from the usual kinematical exchange statistics, though it is a common practice to refer to the net phase simply as 'statistics' without qualification.) 
Composite fermions $(\mathrm{CF})$ are electrons carrying an even number of vortices of the many-particle wave function [5]. In the CF theory, the strongly correlated liquid of interacting electrons at $\nu$ is mapped on to a weakly interacting gas of composite fermions at $\nu^{*}$. The zero-order wave function $\chi_{\nu}$ of interacting electrons at $\nu$ is given by

$$
\chi_{\nu}=\prod_{j<k}\left(z_{j}-z_{k}\right)^{2 m} \Phi_{\nu^{*}}
$$

where $\Phi_{\nu^{*}}$ is a Slater determinant for non-interacting electrons at $\nu^{*}$. The Jastrow factor $\prod_{j<k}\left(z_{j}-z_{k}\right)^{2 m}$ generates $2 m$ vortices in $\chi_{\nu}$ in the relative coordinate of each electron pair, converting the electrons into composite fermions. The LL's of electrons in $\Phi$ translate into the quasi-LL's of composite fermions (after multiplication by the Jastrow factor). The right hand side of Eq. (4) is interpreted as composite fermions at effective filling factor $\nu^{*}$, which is related to the electron filling factor $\nu$ by

$$
\nu=\frac{\nu^{*}}{2 m \nu^{*}+1} .
$$

In particular, the state with $n$ filled quasi-LL's of composite fermions corresponds to the incompressible $\nu=n /(2 m n+1)$ FQHE state. The lowest Landau level projections of these wave functions are known to provide a very good description of the actual eigenstates for systems with small numbers of electrons [7]. For the special case $\nu^{*}=1$, this ground state wave function becomes the original Laughlin wave function [2]. For the purposes of this paper, it will be assumed that various long-distance properties derived with the help of these wave functions are exact.

A related theoretical approach to describing the FQHE states is the introduction of Chern-Simons (C-S) interactions supplementing the electromagnetic interactions. These interactions may be chosen either so that the quasiparticles are fermions [8], in which case the description is equivalent to the CF picture, or so that the quasiparticles are bosons [9, 10], in which case the Laughlin states are described at the first step, and the other FQHE states are obtained in analogy with the Haldane-Halperin hierarchy scheme. Thus the comparisons made here apply also to the C-S approaches even though we do not discuss them explicitly.

\section{QUASIPARTICLES AS DRESSED ELECTRONS}

In the CF theory, it is natural to identify the composite fermions in the topmost partially filled quasi-LL as the objects analogous to the quasielectrons of the QPH scheme. (Equivalently, the holes in the topmost partially filled LL of $\Phi_{\nu^{*}}$ map into the quasiholes of the FQHE state at $\nu$. For simplicity, we restrict our attention to quasielectrons.) Since composite fermions are simply dressed electrons, this identification has two immediate consequences: (i) Quasielectrons have intrinsic charge -e. (ii) Their exchange produces a sign -1 , i.e., they are fermions. These quantities are independent of the specific FQHE state in question. This appears to be in fundamental conflict with the QPH theory, which ascribes a FQHE-state-specific intrinsic charge $-e^{*}$ and fractional statistics $\theta$ to quasiparticles. The objective of this paper is to show 
that despite the apparent dissimilarity, a mean-field treatment of composite fermions provides the same answers for various topological phases as the QPH scheme.

\section{"LOCAL CHARGE" OF QUASIPARTICLES}

A crucial piece of information in the following is the concept of "local charge". While the intrinsic charge of a CF-quasielectron is $-e$, it is screened by the $\mathrm{CF}$ medium, which produces a "correlation hole" around each quasielectron, and the sum of intrinsic charge of the quasielectron and the charge of the correlation hole, measured relative to the charge density corresponding to the background FQHE state, is only a fraction of $-e$. We call this charge the "local charge" of the quasielectron, which can also be defined as the charge in a sufficiently large area containing a quasielectron minus the charge in the same area if it contained no quasielectron. It obviously depends on the screening properties of the background CF state, described by the microscopic wave functions of Eq. (4). There are several ways of obtaining the local charge [2, 3]. We briefly repeat here a derivation that uses a counting argument [11]. Start with the $n /(2 m n+1)$ FQHE state confined to a disk of a given radius, which fixes the largest allowed power of $z_{j}$. Now add an electron to this system while insisting that the size of the system not change. The product in the Jastrow factor in Eq. (画) now goes from 1 to $N+1$, which increases the largest power of $z_{j}$ in the Jastrow factor by $2 \mathrm{~m}$. Therefore, in order to stay within the allotted area, the largest power of $z_{j}$ in $\Phi$ must be reduced by $2 m$, which requires taking $2 m$ electrons from the boundary of each LL and putting them in the interior of the $(n+1)^{\text {st }}$ LL. Including the new electron, the $(n+1)^{s t}$ LL of $\Phi$ now has $2 m n+1$ electrons, i.e., the $(n+1)^{s t}$ quasi-LL of $\chi$ now has $2 m n+1$ composite fermions (or quasielectrons). Since a charge $-e$ was added, each quasielectron has a local charge of

$$
-e^{*}=\frac{-e}{2 m n+1}
$$

which is the same as the intrinsic charge of a quasielectron in the QPH description. Thus, as the added electron gets screened by the medium into a quasielectron of local charge $-e^{*}, 2 m n$ additional composite fermions are excited out of the vacuum. The above argument remains valid even away from a FQHE state, when there are several quasiparticles. The local charge of the quasielectron is still given by $-e^{*}=$ $-e /(2 m n+1)$, where $n$ is the number of quasi-LL's whose edges are occupied. It is worth emphasizing that it is a unique property of an incompressible CF state that the quasiparticle charge is only partially screened (usually, it is either fully screened or not at all), precisely so as to give a simple fractional value for the local charge.

\section{TOPOLOGICAL PHASES}

In the $\mathrm{CF}$ picture, the quasielectron has intrinsic charge $-e$, local charge $-e^{*}$, and obeys fermion exchange statistics. We will be concerned with the phase acquired when a composite fermion (which may be a quasielectron) is taken (counterclockwise) around a closed loop enclosing an area $A$. It has two contributions: one is the usual Aharonov-Bohm $(\mathrm{AB})$ phase, $2 \pi B A / \phi_{0}$, due to a charge $-e$ taken around the loop, 
where $\phi_{0}=h c / e$ is the quantum of flux, and the other contribution comes from the vortices on other composite fermions inside the loop. The total phase is

$$
2 \pi \frac{B A}{\phi_{0}}-4 m \pi K,
$$

where $K$ is the number of composite fermions enclosed in this loop. The second term is a direct consequence of the binding of vortices to electrons, i.e., the formation of composite fermions. This expression for the phase associated with a closed loop is independent of the details of the FQHE state in question.

In a mean-field approximation, similar to the one proposed by Laughlin 12] in his theory of anyons, $K$ is replaced by the average number of composite fermions inside the loop. For the special case of uniform particle density, $\langle K\rangle=A \rho$, where $\rho$ is the particle density per unit area, and the average phase associated with a closed loop is given by

$$
\frac{2 \pi A}{\phi_{0}}\left(B-2 m \rho \phi_{0}\right) .
$$

Therefore, in a mean-field sense the composite fermions move as if they were in an effective field

$$
B^{*}=B-2 m \phi_{0} \rho .
$$

This equation is identical to Eq. (5) with $\nu^{*}=\rho \phi_{0} / B^{*}$ and $\nu=\rho \phi_{0} / B$. In particular, when the effective filling factor of composite fermions is integer $\left(\nu^{*}=n\right)$ the corresponding electron state at $\nu=n /(2 m n+1)$ is incompressible, resulting in the FQHE.

In the QPH description, the quasielectron is assumed to have intrinsic charge $-e^{*}$, local charge also $-e^{*}$, bosonic exchange statistics, and fractional anyon statistics $\theta$. Below we show, in several examples, that this description also produces the same answer for the phases as the mean-field approximation of Eq. (7). We find it convenient to use a slightly different convention for the statistics of the QPH-quasiparticles. We assume that they obey fermionic exchange statistics and anyon statistics $\theta^{*}$ [13], where

$$
\theta^{*}=\theta-1=-\frac{2 m}{2 m n+1} .
$$

The total statistics, including in a single phase factor both the exchange statistics and the phase coming from the dynamical interaction, is $\theta$ in either convention.

One quasiparticle: Consider a system with only one quasielectron, i.e., only one composite fermion in an otherwise empty quasi-LL, with the lower $n$ quasi-LL's fully occupied. Let the quasielectron go around a closed loop in the counterclockwise direction. The average phase associated with this path is $2 \pi B^{*} A / \phi_{0}$. In the $\mathrm{QPH}$ picture, the phase associated with this path is $2 \pi B A / \phi_{0}^{*}$ where

$$
\phi_{0}^{*}=\frac{h c}{e^{*}}=(2 m n+1) \phi_{0} .
$$


The two are equal since $B^{*}=B /(2 m n+1)$ for incompressible states (or, $e B^{*}=$ $e^{*} B$ ). The $\mathrm{CF}$ result would be obtained if the Berry phase calculation of Ref. [6] is interpreted in terms of an effective magnetic field rather than a fractional intrinsic charge.

Two quasiparticles: Now consider two quasielectrons, and let one go around a closed loop in the counterclockwise direction. The average phase is $2 \pi B A / \phi_{0}-4 m \pi \rho A$ when this loop does not enclose the other quasielectron, and $2 \pi B A / \phi_{0}-4 m \pi(\rho A+$ $\left.e^{*} / e\right)$ when it does. The difference between the two is $2 \pi \theta^{*}$ with

$$
\theta^{*}=-2 m \frac{e^{*}}{e}
$$

in agreement with the anyon statistics, Eq. (10), of the QPH theory. This is a "derivation" of anyon statistics starting from the CF theory. Note that the mean-field approximation of averaging over positions of other composite fermions is necessary for obtaining fractional statistics (which entails non-analyticity); for any given configuration of other composite fermions, the phase due to vortices is always a multiple of $2 \pi$.

Several quasiparticles: Away from the special filling factors, when there is a finite density of quasiparticles, assumed to be uniformly distributed, the average phase associated with a closed loop is still given by Eq. (8) according to the CF theory. This result is obtained in the QPH theory as follows. Consider a magnetic field $B<B_{0}$ where

$$
B_{0}=\frac{2 m n+1}{n} \rho \phi_{0}
$$

corresponds to the FQHE state at $\nu_{0}=n /(2 m n+1)$. The quasielectron density per unit area at $B$ is given by

$$
\rho_{q}=n \frac{\left(B_{0}-B\right)}{\phi_{0}} .
$$

(This follows since each flux quantum away from $B_{0}$ produces $n$ quasiparticles.) The average phase associated with a closed loop is then given by

$$
2 \pi A\left(\frac{B}{\phi_{0}^{*}}+\rho_{q} \theta^{*}\right)
$$

which is the same as Eq. (8).

Resonant tunneling: Consider next a resonant tunneling situation in which a composite fermion tunnels from one edge of the sample to the other through a path that goes around a potential hill containing no electrons. According to Eq. (7), with $K=0$, the phase associated with this path is simply the usual AB phase

$$
2 \pi \frac{B A}{\phi_{0}},
$$


which implies that successive resonant tunneling peaks are expected when the flux through the electron-free region changes by $\phi_{0}$. This period was anticipated theoretically [14] and has also been observed experimentally [15, 16]. Note that the charge deficiency of the electron-free region is an integer multiple of the local charge $e^{*}$, as observed experimentally [15]. In the quasiparticle picture, if we assume that the quasiparticles, their fractional charge and their fractional statistics are well defined at the boundary of the FQHE state (which is a non-trivial assumption due to the absence of a gap there), and also recognize that the electron-free region is made of $N_{q}=n A B / \phi_{0}$ quasiholes, then the phase is given by

$$
2 \pi \frac{B A}{\phi_{0}^{*}}+2 \pi N_{q}\left(-\theta^{*}\right)
$$

where $-\theta^{*}$ is the relative statistics of quasielectron and quasihole. This also reduces to the previous equation.

Spherical geometry: Interpretation by fractionally-charged fractional-statistics quasiparticles becomes more complicated in spherical geometry [4, where the electrons move on the surface of a sphere under the influence of a radial magnetic field. In this geometry, the electron system at flux $N_{\phi}$ is mapped on to the CF system at flux

$$
N_{\phi}^{*}=N_{\phi}-2 m(N-1)
$$

which reduces to Eq. (17) in the limit of large $N$, with $\nu=N / N_{\phi}$ and $\nu^{*}=N / N_{\phi}^{*}$. This equation has been tested in numerical work on small systems, which have shown a striking similarity between the low-energy spectrum of interacting electrons at $N_{\phi}$ and that of noninteracting fermions at $N_{\phi}^{*}$ in a broad range of filling factors [7]. For a single composite fermion in the $n$th quasi-LL (with $n=1$ being the lowest quasi-LL), the phase associated with a closed path is

$$
L^{*} \Omega
$$

where $L^{*}=\frac{N_{\phi}^{*}}{2}+n-1$, and $\Omega$ is the solid angle of the path. In the quasiparticle picture, it would be natural to write the analogous phase as [17]

$$
2 \pi \frac{e^{*}}{e} \frac{\Omega}{4 \pi} N_{\phi}
$$

which differs from the previous equation by a term of order unity. This term has been interpreted as a new quantum number of the quasiparticle, the "spin" [17. (It has nothing to do with the actual spin of the electron. The electrons have been assumed to be spinless in this work, as appropriate for a fully polarized system of electrons.) The expression for the spin is, however, rather complex, and does not satisfy any simple relation with the anyon statistics of the quasiparticles. In particular, the quasiparticle and the quasihole spins are not equal in magnitude. In agreement with 
an earlier suggestion [18], Einarsson et al. [17] have shown that the part of the spin which is even under charge conjugation (i.e., the average of the quasielectron and quasihole spins) is well defined and satisfies the usual connection with the fractional statistics $\theta^{*}$.

\section{EFFECTIVE ELECTRIC FIELD}

An effective 'renormalization' of the magnetic field implies an effective 'renormalization' of the electric field due to the following argument. Assume a uniform electric field $E$ in the plane of the electron system, so electrons move with velocity $v=c E / B$ in a direction transverse to $E$. This result is independent of the nature of the interaction-induced correlations, most easily seen by going to a new frame of reference moving with velocity $v$, where there is no electric field. Therefore, composite fermions, which experience an effective magnetic field $B^{*}$ rather than $B$, must also experience an effective electric field $E^{*}$, satisfying

$$
\frac{E^{*}}{B^{*}}=\frac{E}{B}
$$

i.e.,

$$
E^{*}=E-2 m \rho \phi_{0} \frac{E}{B} .
$$

As in Eq. (8), the second term on the right hand side is induced by the vortices carried by electrons, as explained in Ref. [10] in the context of the boson ChernSimons theory. We repeat the argument briefly. Consider the current e $\rho v W$ through a cross section of width $W$. Since each electron carries with it $2 m$ vortices, there is also a vortex current given by $2 m \phi_{0} \rho v W$. This, by Faraday's law, leads to a potential $2 m \phi_{0} \rho v W / c$ across this region of width $W$, thereby providing the second term in the above equation. For the incompressible state at $\nu=n /(2 m n+1)$, the effective electric field is given by $E^{*}=\left(e^{*} / e\right) E$, since $E^{*} / E=B^{*} / B=\nu / \nu^{*}=1 /(2 m n+1)=e^{*} / e$.

The concept of effective electric field helps resolve an apparent problem with the interaction energy of two quasiparticles within the CF scheme. The CF picture for the quasielectron is somewhat reminiscent of an electron in a dielectric, which is screened by electric dipoles to produce a local charge $-e_{L}$. The Coulomb energy of two electrons embedded in the dielectric, at a distance $r$, is $e_{L} e / r$, which is equal to the work done in bringing an electron from infinity to $r$ under the influence of the electric field of the charge $-e_{L}$ object at the origin. The coupling of the incoming electron with the electric field is with the full charge $-e$, rather than the local charge $-e_{L}$. The same is true of coupling with the magnetic field, as required by the gauge invariance of electrodynamics. Thus, the screening which reduces the local charge has no effect on the coupling with the electromagnetic field, a circumstance which is true much more generally for electrons in complex media [19. Now let us consider the $\nu=n /(2 m n+1)$ FQHE state. The quasielectrons of this state experience the magnetic field $B$ with the full charge $-e$, as implicit in Eq. (7). By gauge invariance, this can only be true if the coupling to an electric field also entails the same charge 
$-e$. Naively, i.e, without taking account of the electric field renormalization, this would suggest that the interaction energy of two FQHE quasielectrons is $e^{*} e / r$. (For the discussion here, we assume that the FQHE system is in vacuum, rather than being at the interface of two dielectrics.) This is in contradiction with the correct result, known to be $\left(e^{*}\right)^{2} / r$ [2]. However, the interaction energy with the effective electric field is given by $\left(e^{*} / e\right) e^{*} e / r$, reproducing the correct result.

\section{DISCUSSION}

It might seem surprising that the two approaches obtain the same answers for the long range interactions, both electric and magnetic, of quasiparticles, starting from two rather different viewpoints. However, this happens because the very assumption of incompressibility at a fractional filling factor puts strong constraints on these quantities. In fact, $\mathrm{Su}$ [20] has shown that the long-range electromagnetic interactions of the quasiparticles are determined uniquely for any given incompressible FQHE state, provided one assumes that there is only one type of quasiparticle, which carries the largest allowed local charge.

The QPH theory is formulated in terms of the quasielectrons, i.e., the composite fermions of the topmost quasi-LL. The CF theory on the other hand treats all composite fermions in an equivalent fashion. The full treatment in terms of composite fermions of various quasi-LL's becomes essential in the limit of large $n$. Here, the gap between neighboring quasi-LL's disappears and it is not meaningful to describe the dynamics in terms of fractionally-charged fractional-statistics quasiparticles, but the CF description may, in principle, continue to be valid. In an insightful work, Halperin, Lee, and Read [21] interpreted certain experimental anomalies near $\nu=1 / 2$, where no FQHE is observed, as a signature of the existence of a Fermi sea of composite fermions. Several subsequent experiments have produced evidence for composite fermions and their Fermi sea in the vicinity of $1 / 2$ [22]. Note that, unlike for the incompressible FQHE states, the local charge of the CF-quasiparticle, and consequently also its anyon statistics are not well defined for the the compressible $\nu=1 / 2$ liquid, due to the absence of a gap [19], but its intrinsic charge $-e$ and fermion exchange statistics are still sharp observables. The observed cyclotron radius at $\nu=1 / 2$ is consistent with the existence of a Fermi surface of charge $-e$ fermions, and provides direct evidence for the intrinsic quantum numbers of composite fermions. The Shubnikov-de Haas analysis of Ref. [23], and the explanation of the thermopower measurements of Ref. [24] also assume charge $-e$ fermions.

In conclusion, we have analyzed two descriptions for the quasiparticles of the FQHE, one in which they are viewed as novel, topological objects with intrinsic fractional electric charge and intrinsic fractional statistics, and the other in which they are pictured as composite fermions, i.e., as dressed electrons. The CF theory has the pleasing aspect that the quasielectrons are the same composite fermions as those in the lower quasi-LL's, in other FQHE states, or even in the compressible Fermi liquid state. Furthermore, it reveals that a quasielectron is simply an electron dressed

by the composite fermion medium, and hence has the same intrinsic charge, spin and 
exchange statistics as an electron, independent of the background state. Its local charge, on the other hand, depends on the screening properties of the background CF state. This seems to be very much in the spirit of quasiparticle descriptions used for many other interacting electron systems in condensed matter physics. The CF theory thus unifies the view of quasiparticle dynamics in and even beyond the FQHE

regime. The remarkable new physics of the FQHE arises from the special nature of correlations which provide additional phases, leading to what may be a unique phenomenon, the dynamical renormalization of the electromagnetic field.

We have benefited greatly from extensive discussions with Steven Kivelson. Torbjorn Einarsson, Hans Hansson and Jon-Magne Leinaas also made instructive comments. This work was supported in part by the National Science Foundation under grant numbers PHY93-09888 (ASG) and DMR93-18739 (JKJ).

\section{References}

[1] D.C. Tsui, H.L. Stormer, and A.C. Gossard, Phys. Rev. Lett. 48, 1559 (1982).

[2] R.B. Laughlin, Phys. Rev. Lett. 50, 1395 (1983).

[3] B.I. Halperin, Phys. Rev. Lett. 52, 1583 (1984).

[4] F.D.M. Haldane, Phys. Rev. Lett. 51, 605 (1983). Note that fractional statistics does not appear explicitly in Haldane's construction.

[5] J.K. Jain, Phys. Rev. Lett. 63, 199 (1989).

[6] D.P. Arovas, J.R. Schrieffer, F. Wilczek, Phys. Rev. Lett. 53, 722 (1992).

[7] G. Dev and J.K. Jain, Phys. Rev. Lett. 69, 2843 (1992); X.G. Wu, G. Dev, and J.K. Jain, Phys. Rev. Lett. 71, 153 (1993).

[8] A. Lopez and E. Fradkin, Phys. Rev. B 44, 5246 (1991).

[9] S.M. Girvin and A.H. MacDonald, Phys. Rev. Lett. 58, 1252 (1987).

[10] S.C. Zhang, H. Hansson, S.A. Kivelson, Phys. Rev. Lett. 62, 82 (1989); S.C. Zhang, Int. J. Mod. Phys. B 6, 25 (1992).

[11] J.K. Jain, Comments Cond. Mat. Phys. 16, 307 (1993).

[12] R.B. Laughlin, Phys. Rev. Lett. 60, 2677 (1988).

[13] This would be the result of the Berry phase calculation of Ref. [6 if the wave function for two quasiholes at $\eta$ and $\eta^{\prime}$ is taken to be $\left(\eta-\eta^{\prime}\right) \prod_{j}\left(z_{j}-\eta\right)\left(z_{j}-\eta^{\prime}\right) \chi_{L}$, as opposed to the one without the factor $\left(\eta-\eta^{\prime}\right)$. 
[14] S. Kivelson, Phys. Rev. Lett. 27, 3369 (1990); P.A. Lee, ibid. 65, 2206 (1990); J.K. Jain, S.A. Kivelson, and D.J. Thouless, Phys. Rev. Lett. 71, 3003 (1993).

[15] V.J. Goldman et al., Science, in print.

[16] C.J.B. Ford et al., J. Phys. Cond. Mat. 6, L725 (1994).

[17] T. Einarsson, S.L. Sondhi, S.M. Girvin, and D.P. Arovas, preprint; also see, X.G. Wen and A. Zee, Phys. Rev. Lett. 69, 953 (1992); ibid. 3000 (1992) [E]; D.-H. Lee and X.-G. Wen, Phys. Rev. B 49, 11066 (1994).

[18] A.S. Goldhaber and R. MacKenzie, Phys. Lett. B214, 471 (1988); A.S. Goldhaber, unpublished.

[19] For further discussion, see A.S. Goldhaber and S.A. Kivelson, Phys. Lett. B255, 445 (1991).

[20] W.P. Su, Phys. Rev. B 34, 1031 (1990).

[21] B.I. Halperin, P.A. Lee, and N. Read, Phys. Rev. B 47, 7312 (1993).

[22] R.L. Willett et al., Phys. Rev. Lett. 71, 3846 (1993); W. Kang et al., Phys. Rev. Lett. 71, 3850 (1993); V.J. Goldman et al., Phys. Rev. Lett. 72, 2065 (1994).

[23] D.R. Leadley, et al., Phys. Rev. Lett. 72, 1906 (1994); R.R. Du et al., Solid State Commun. 90, 71 (1994).

[24] X. Ying, V. Bayot, M.B. Santos, and M. Shayegan, Phys. Rev. B 50, 44969 (1994). 